\title{
THE INSURER'S RUIN
}

\author{
B. H. DE JONGH
}

Amsterdam

\section{INTRODUCTION}

This paper was written in connection with the preparation of Marché Commun regulations in the insurance sector and has been submitted to the Commission Technique pour l'étude d'un indice de solvabilité relatif aux entreprises d'assurances contre les dommages. It aims at explaining the scope of the problem to non-mathematicians and for that reason emphasizes its logical in contradistinction to its computational aspects.

Sect. I

The probability of the insurer's ruin has two aspects. The occurrence of the event ruin may be considered with respect to a fixed period but also with respect to a period of undetermined length. In both cases the period starts at a moment at which the insurer's capital (patrimoine) is known and it is intuitively clear that in both cases the probability of ruin will be the higher as the insurer's capital is smaller and the risk to which he is exposed heavier.

For some purposes more precise conclusions are required. This requirement gives rise to problems which will be considered here with respect to the probability of the occurrence of ruin in a period of undetermined length. It will be taken for granted that besides the artificial events occurring in games of chance there are other classes of uncertain events to which numerical probabilities can be assigned and that, as far as claims are concerned, the insurer's losses belong to one of the said classes. On this understanding it makes sense to consider a numerical probability of ruin depending on a fixed initial capital, random losses to which numerical probabilities are assigned, and other profits and losses.

In this connection it is reasonable to consider random losses after deduction of the random amounts covered by reinsurance. 
The two random elements "losses" and "recovered losses" can of course also be considered separately. If, as is sometimes suggested, the random amounts which are covered by reinsurance are left out of consideration it is not possible to arrive at a numerically determined probability of ruin in accordance with the insurer's actual risk.

As far as the random losses are concerned the solution of the resulting problem has a logical as well as a mathematical aspect. The logical aspect is perhaps unfamiliar but it is essentially simple. Consider the event $R$ (= ruin, occurring at an undetermined future moment) and its decomposition into the following mutually exclusive events. For the benefit of the reader who is not familiar with the symbolism appropriate to this occasion, the decomposition is written down in full:

Either $\left\{\begin{array}{l}\text { arrival from initial } \\ \text { capital } z \text { at the } \\ \text { capital } x \leq 0 \text { in the } \\ \text { first unit time- } \\ \text { interval }\end{array}\right\}$

or $\left\{\left(\begin{array}{l}\text { arrival from } \\ z \text { at } x=\mathrm{I} \\ \text { in the first } \\ \text { unit time- } \\ \text { interval }\end{array}\right)\right.$ as $\left.\begin{array}{c}\text { aell } \\ \text { as }\end{array}\left(\begin{array}{l}\text { arrival from } x=\mathrm{I} \\ \text { at } x \leq \mathrm{o} \text { at the end } \\ \text { of a subsequent } \\ \text { time-interval of } \\ \text { undetermind length }\end{array}\right)\right\}$

or $\left\{\left(\begin{array}{l}\text { arrival from } \\ z \text { at } x=2 \\ \text { in the first } \\ \text { unit time- } \\ \text { interval }\end{array}\right)\right.$ as $\left.\begin{array}{c}\text { asll } \\ \text { as }\end{array}\left(\begin{array}{l}\text { arrival from } x=2 \\ \text { at } x \leq \text { o at the end } \\ \text { of a subsequent time } \\ \text { interval of undeter- } \\ \text { mined length }\end{array}\right)\right\}$

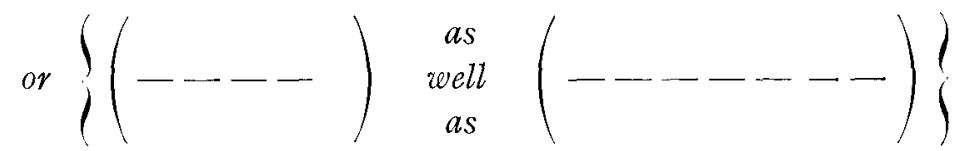

This logical decomposition leads to a set of equations in terms of probabilities of events and hence to the mathematical problem 
how to solve the equations. An extensive literature on this subject is available. ${ }^{1}$ ) In this paper W. Feller's method will be followed; it uses less advanced mathematics than others.

Feller obtains a mathematical simplification by taking account of a second restraint besides the fixed initial capital $z$. It is assumed that the risk process comes to an end as soon as the capital reaches the amount $a \geqslant z$. In games of chance this assumption comes natural. They come to an end as soon as the adversary's capital is absorbed. From a logical point of view this apparent complication is quite welcome. It means that the above decomposition has a finite number of terms. Its last term then refers to the arrival from $z$ at $a-\mathrm{I}$ in the first unit time interval. With respect to a discussion of the insurer's ruin the constant $a$ enters only by way of a preliminary; it can and will be removed at the end of the argument.

Sect. 2

The decomposition discussed in Sect. I can be written in the form

$$
\begin{gathered}
R_{u}^{(z)}=R_{\perp}^{(z)} \cup\left\{A_{1}^{x=1} \cap R_{u}^{(1)}\right\} \cup\left\{A_{1}^{(x=2)} \cap R_{u}^{(2)}\right\} \cup \ldots \\
\ldots \ldots \cup\left\{A_{1}^{(x=a=1)} \cap R_{u}^{(a-1)}\right\}
\end{gathered}
$$

The probability of the event $R_{u}^{(z)}$ equals the probability of its decomposition. The latter can be expressed in terms of probabilities of the single events specified on the right hand side of (I). Remembering that the probability of the " $u$ " (三union) of mutually exclusive events equals the sum of their probabilities we obtain

$$
p\left(R_{u}^{(z)}\right)=p\left(R_{1}^{(z)}\right)+\sum_{x-1}^{a-1} p\left\{A_{1}^{x} \cap R_{u}^{x}\right\}
$$

The terms $A_{i}^{x}, R_{u}^{x}$ within brackets refer to events which are assumed to be statistically independent. Hence the probability of their " $n$ " ( $\equiv$ joint) equals the product of their probabilities.

1) Some of the first publications on the subject are by F. Lundberg (1926), H. Cramér (1930), C. O. Segerdahl (1939), B. de Finetti (1939). Further reference is made to J. Dubourdieu, Théorie Mathématique du Risque, Paris 1952 and W. Feller, Probability Theory and its Applications, New YorkLondon 1952.

A comprehensive survey of the theory is given by $\mathrm{H}$. Cramér, Collective Risk Theory, Stockholm I955. 
Accordingly

$$
p\left(R_{u}^{(z)}\right)=p\left(R_{1}^{(z)}\right)+\sum_{x-\mathrm{I}}^{a-1} p\left(A_{1}^{x}\right) \cdot p\left(R_{u}^{x}\right)
$$

or in a modified notation

$$
r_{u}(z)=r_{1}(z)+\sum_{x=1}^{a-1} \tilde{p}_{1}(x-z) r_{n}(x)
$$

We note that the event $A_{1}^{x}$ refers to the arrival from $z$ at $x$ at the end of the first unit-time interval. This event is equivalent to the event that, over the same time interval, the capital's increment equals $x-z$. In the notation of (4) this increment is made explicit. The term increment also refers to non-positive mutations.

By (4) one obtains a system of equations consisting of one equation for every value of $z=\mathrm{I}, 2, \ldots a-\mathrm{I}$. The function $\tilde{p}_{1}($. being given, the system has a solution which uniquely determines the unknown function $r_{u}($.$) .$

The solution of (4) is a purely mathematical matter. It will be discussed in Sect. 4. In Sect. 3 the nature of the capital's random increment over one time interval will be discussed.

Sect. 3

In practice the capital's random increment, that is the insurer's random gain over the unit time-interval, contains besides random losses (in the sense of claims, claims recovered by reinsurance being deducted) ordinary profits and losses. In order to simplify the argument we consider the gain as a random variable $g$ such that

$$
\underline{g}=-|\underline{s}|+(I+\beta) E|\underline{s}|
$$

where $s$ represents the total random loss in monetary units occurring over the unit time-interval after deduction of the random amount covered by reinsurance.

All other profits and losses are, by means of the constant factor $I+\beta$, expressed in terms of the expectation value of $|s|$.

It is of course possible to devise a more elaborate and more realistic analysis of the insurer's gain $\underline{g}$. In this connection reference is made to J. Dubourdieu (ibid. V, II). A more refined analysis will however not lead to a different logical structure of the argu- 
ment and for that reason it will be omitted here. It is intuitively clear that, if in (5) the constant $\beta$ is negative, future ruin is certain. The case $\beta>0$ which implies that $E g>0$ and the case $\beta=0$ which implies that $E g=0$ will be discussed in the next section.

Sect. 4

Equation (4) may be written in the form of the system

$$
r_{u}(z)=\sum_{-\infty}^{\infty} r_{u}(x) \tilde{p}_{1}(x-z)
$$

subject to the $\quad r_{u}(x)=$ I if $x \leq 0$

boundary conditions: $r_{u}(x)=0$ if $x \geq a$

where $\tilde{p}_{1}($.$) is the probability distribution of the capital's increment$ $\underline{x}-z=\underline{g}$ during one time interval. In principle an exact solution of (6) can be found by means of the roots of the characteristic equation

$$
\sum p_{1}(g) s^{g}=\mathrm{I}
$$

This method works when the increment $g$ takes only a small number of values. If, as practically always, this number is large an approximate solution can be found. It can be shown that if $E \underline{g}>0$ or, equivalently, the constant $\beta$ in (5) is positive equation (7) has, besides the positive root $s=I$, one and only one other positive root $\tilde{s}$, say, which is smaller than $\mathbf{I}$.

Then (cf. W. Feller, Prob. Th. and Appl. I4.8) the following unequalities hold ${ }^{1}$ ):

$$
\frac{\tilde{s}^{a}-\tilde{s}^{z}}{\tilde{s}^{a}-\tilde{s}^{-\nu+1}} \leqslant r_{u}(z) \leqslant \frac{\tilde{s}^{a+\nu^{*}-1}-\tilde{s}^{z}}{\tilde{s}^{a+\nu^{*}+1}-\mathrm{I}}
$$

where $g=x-z$ takes the values $-\nu, \longrightarrow \nu+\mathbf{I}, \ldots, \nu^{*}-\mathbf{I}, \nu^{*}$.

It has been pointed out in Sect. I that with respect to the insurer's ruin the presence of the constant $a$ has no sense. It can now be removed by letting $a \rightarrow \infty$. Accordingly (8) takes the simple form.

$$
\tilde{s}^{z+v-1} \leqslant r_{u}(z) \leqslant \tilde{s}^{z} \quad \beta>0
$$

1) For the purpose of checking the following inequalities some data are given in the appendix. 
Further, if $\beta<0$, the second positive root of equation $(7)$ is larger than $I$.

It then follows that

$$
r_{u}(z)=\mathrm{I} \quad \beta<0
$$

The certaintly of the occurrence of ruin at an undetermined time, as expressed by (Io) for the case that $\beta<0$ also holds if $\beta=0$.

In that case (8) takes a simpler form.

Sect. 5

It should be noted that the numerical reliability of the results (8), (9), (Io) depends on the reliability of the hypothesis which assigns to the insurer's gain (5) a particular distribution $\tilde{p}_{1}(g)$. This reserve does not particularly apply to the calculation of the probability of ruin occurring at an undetermined time; exactly the same hypothesis is required if the probability of ruin occurring in a fixed time has to be determined.

The results obtained are useful if the probability of the insurer's ruin has to be considered from a general point of view. The simple conclusion:

"if no surcharge $\beta$ is earned and accumulated ruin is certain" is of importance with respect to fiscal questions. With respect to individual companies and their solvency one is faced with the problem how to justify a particular distribution with respect to the company's gain.

On this subject the writer takes the view that if for some purpose (e.g. solvency standards in the Common Market) a definite answer to this question is required this cannot be given before an extensive statistical study has been made and that it is by no means sure that as a result of this study a workable system will be obtained. There are, however, simpler ways for dealing with the problem of an insurance company's solvency and its supervision. 


\section{Appendix}

It can be shown (cf W. Feller, Prob. Th. and Appl. I4.8) that if in (5) the constant $\beta$ is positive the equation

$$
\Sigma \check{p}_{1}(g) s^{g}=\mathbf{I}
$$

has exactly 2 positive roots, one of which equals I and the other, to be denoted by $\tilde{s}$ is smaller than $\mathbf{I}$.

Then

$$
r_{u}(z)=A+B \tilde{s}^{z}
$$

A. I

satisfies (6) but, in general, not the boundary conditions (6)

$$
\text { Check: } \begin{aligned}
r_{u}(z) & =\sum r_{u}(x) \tilde{p}_{1}(x-z) \\
A+B \tilde{s}^{z} & =\Sigma\left(A+B \tilde{s}^{x}\right) \tilde{p}_{1}(x-z) \\
& =A \Sigma \tilde{p}_{1}(x-z)+B \Sigma \tilde{s}^{x} \tilde{p}_{1}(x-z) \\
& =A \Sigma \tilde{p}_{1}(g)+B \Sigma \tilde{s}^{g}+z \tilde{p}_{1}(g) \\
& =A+B \tilde{s}^{z}
\end{aligned}
$$

An approximate solution of $r_{u}(z)$ which satisfies (6) and also the boundary conditions is obtained as follows

I. Let $g$ be defined on $\left(-v, v^{*}\right)$. Owing to the first boundary condition of (6) the terms referring to $x \leq 0$ add to $r_{1}(z)$. Example for $-v=-3$ :

$$
\begin{gathered}
r_{u}(-3) \tilde{p}_{1}(-3-z)+r_{u}(-2) \tilde{p}_{1}(-2-z)+ \\
+r_{u}(-\mathrm{I}) \tilde{p}_{1}(-\mathrm{I}-z)+r_{u}(\mathrm{o}) \tilde{p}_{1}(z)
\end{gathered}
$$

Terms containing the factor $r_{u}(x), x \leq-v$, are cancelled as the factor $\tilde{p}_{1}(x-z)$ vanishes if $x \leq v$. Hence the first boundary condition is equivalent to

$$
r_{u}(x)=\mathrm{I} \text { if }-v<x \leqslant 0
$$

A. 2

By the second boundary condition of (6) all terms referring to $x \geqslant a$ vanish. They vanish a fortiori if $x \geqslant a+v^{*}$. As, by definition, $a>z$ it then follows that

$$
x-z \geqslant a+v^{*}-z>v^{*}
$$


and in that case the factor $\tilde{p}_{1}(x-z)$ vanishes. Accordingly the second boundary condition of (6) is equivalent to

$$
r_{u}(x)=0 \text { if } a \leqslant x \leqslant a+v^{*}
$$

A. 3

2. Put

$$
\left.\begin{array}{l}
A+B \tilde{s}^{a+v^{*}-1}=0 \\
A+B \tilde{s}^{0}=\mathrm{I}
\end{array}\right\} \begin{aligned}
& A=\frac{-\tilde{s}^{a+v^{*}-1}}{\mathrm{I}-\tilde{s}^{a+v^{*}-1}} \\
& B=\frac{\mathrm{I}}{\mathrm{I}-\tilde{s}^{a+v^{*}-1}}
\end{aligned}
$$

It then follows that

$r_{u}(x)=A+B \tilde{s} x=\frac{\tilde{s}^{x}-\tilde{s}^{a+v^{*}-1}}{\mathrm{I}-\tilde{s}^{a+v^{*}-1}} \geqslant\left\{\begin{array}{l}\mathrm{I} \ldots \quad x \leqslant 0 \\ 0 \ldots a \leqslant x<a+v^{*}\end{array}\right.$

By (5) and A2 $v_{u}(z)$ must satisfy the condition

$$
r_{u}(z)=\left\{\begin{array}{l}
\text { I } \ldots \quad z \leqslant 0 \\
0 \ldots a \leqslant z \leqslant a+v^{*}
\end{array}\right.
$$

It then follows that

$$
r_{u}(z) \leq \frac{\tilde{s}^{a+v^{*}-1}-\tilde{s}^{z}}{\tilde{s}^{a+v^{*}-1}-I}
$$

3. Put

$$
\begin{aligned}
& A+B \tilde{s}^{a} \quad=0 \quad A=\frac{\tilde{s}}{\tilde{s}^{a}-\tilde{s}^{-v+1}} \\
& \left.A+B \tilde{s}^{-v+1}=\mathbf{I}\right\} \quad B=\frac{-\mathrm{I}}{\tilde{s}^{a}-\tilde{s}^{-\nu+1}}
\end{aligned}
$$

It then follows that

$$
A+B \tilde{s}^{x}=\frac{\tilde{s}^{a}-\tilde{s}^{x}}{\tilde{s}^{a}-\tilde{s}^{-\nu+1}} \leqslant\left\{\begin{array}{l}
\mathrm{I} \ldots-\nu<x \leqslant 0 \\
0 \ldots a \leq x
\end{array}\right.
$$

$\mathrm{By}(5)$ and $3 \mathrm{~A} r_{u}(z)$ must satisfy the condition $r_{u}(z)=\left\{\begin{array}{l}\mathrm{I} \ldots-v<z \leqslant 0 \\ 0 \ldots a \leqslant z\end{array}\right.$

It then follows that $r_{u}(z) \geq \frac{\tilde{s}^{a}-\tilde{s}^{z}}{\tilde{s}^{a}-\tilde{s}^{-v+1}}$

A. 5 
4. Combining $\mathrm{A}_{4}$ and $\mathrm{A}_{5}$ it follows that

$$
\frac{\tilde{s}^{a}-\tilde{s}^{z}}{\tilde{s}^{a}-\tilde{s}^{-v+1}} \leqslant r_{u}(z) \leqslant \frac{\tilde{s}^{a+v^{*}-1}-\tilde{s}^{z}}{\tilde{s}^{a+v^{*}-1}-\mathrm{I}} \quad \text { A. } 6
$$

on the understanding that $\beta>0$. The same relation holds if $\beta<0$. The following remark is of theoretical interest only: if $v=\nu^{*}=\mathrm{I}$ the inequalities $\mathrm{A} 6$ reduce to an equality. 